(c) Journal of Applied Mathematics \& Decision Sciences, 1(2), 133-150 (1997)

Reprints Available directly from the Editor. Printed in New Zealand.

\title{
Combination Trading with Limit Orders ${ }^{*}$
}

\author{
HENRY SCHELLHORN \\ hschellh@us.oracle.com \\ Oracle Corporation, 604 Arizona Avenue, Santa Monica, CA 90401 USA.
}

\begin{abstract}
We model the exchange of commodities that are contingent upon each other, when traders place mostly limit orders. Examples include: 1) a market of financial futures where future spreads are also traded, 2) a market of mutual funds and stocks, 3) a market of options and stocks, under the viewpoint that they are both combinations of Arrow-Debreu securities. We prove that consistent prices are optimal. We develop a fixed-point algorithm to compute an optimal price and allocation. The algorithm combines ideas from contraction mapping theory and from homotopy theory. It is much faster than a traditional linear programming approach.
\end{abstract}

Keywords: Economic equilibrium computation, equilibrium programming, homotopy, auction.

\section{Introduction}

Due to the progress of automation, options and futures exchanges have recently expanded the possibility to trade various combinations of primitive financial instruments. Among others, the Chicago Mercantile Exchange, the German and the Swiss options and futures exchanges all offer standardized future time spreads contracts, where a potential buyer buys one leg (the future with early expiration) and sells the other (the future with later expiration) at the same time and delivers or receives money for this trade.

This is only one example of combination trading. More generally, a combination can be described in terms of the coefficients of primitive instruments it includes. For example, the coefficient of a future time spread as above or of a swap would be $[1,-1]$. A mutual fund is a combination with only positive but maybe fractional coefficients. Options are themselves combinations, where the primitive instruments are Arrow-Debreu securities.

We focus on double auction markets, where buyers and sellers place limit orders, i.e., orders for a predetermined quantity at a predetermined price. As in markets without combinations, our model allows for a certain quantity of market orders. Currently, options and futures exchanges are primarily auction markets. We do not discuss the relative merits of dealer markets compared to continuous or batched auctions. This question is still an open issue in the literature. Among others, [7] reports that batched auctions are more robust mechanisms, and therefore may be

$†$ Correspondence to: Henry Schellhorn, 525 N. Gardner Avenue, Los Angeles, CA 90036, USA. * This work was sponsored by a grant from the Swiss National Fund for Scientific Research. I acknowledge the help of my Ph.D. committee at UCLA, composed of Professors N. Bambos, S. Jacobsen, B. Miller, E. Schwartz, and K. Yao. All errors are mine. 
preferable in very illiquid markets. A continuous auction mechanism involving combinations, also called synthetics is described in [8].

Combinations are especially useful in auction markets because of the liquidity risk inherent to auctions. For instance, a trader holding a Swiss Franc future contract expiring in June may suddenly want to swap it against the same contract expiring in July. The reason why he would prefer to place a combination order than two independent orders is that he much prefers his current position to

- either a null position due to a successful sale and the absence of counterparty that would sell the July contract

- or a "doubled position" due to the absence of a counterparty that would buy the June contract.

Combination trading can be seen as a practical compromise between the classical tatonnement process and the so-called "equilibrium process", introduced by Beja and Hakansson in [1], where "all traders[..] submit [..] a complete specification of tentative orders for all possible prices". An equilibrium process gives traders greater flexibility. However, according to the same authors, "the (implicit) cost of placing orders makes these requirements prohibitively expensive". Wohl [11] criticizes most existing markets where "an order in one security cannot be conditioned on prices of other securities", and describes a mechanism that implements a simplified equilibrium process, where an order in one security is conditioned on the prices of the same security and of an index.

A simple strategy allows traders to implement Wohl's conditioned orders ${ }^{1}$ in a combination trading exchange. The main advantages of the "combination trading" approach to implement the conditioned orders over the approach described in [11] are that traders can condition their orders on all other securities and that the equilibrium is realized in discrete prices. We have also been able to verify [9] that, like in [11], our analysis applies to more general types of limit orders than described in section 2 .

The main purpose of this paper is to examine how the traders' orders for combinations can be matched optimally with each other and with other traders' orders for primitive instruments. The role of the exchange institution is to provide a discrete price vector and an allocation that maximizes the utilitarian social welfare functional. We show that this problem reduces to solving the so-called market balance problem, in which commodity prices are consistent. The proof of consistency relies on standard duality techniques; it has been a subject in literature before (e.g. [10]) for a simple market without combinations.

We suggest two different algorithms to solve the market balance problem and compare them against the only possible method to solve the combination trading problem, namely linear programming. Although special instances of combination trading can be formulated as a network flow problem of a manageable size, the general problem cannot. 
When there is a large number of orders, the aggregate demand and supply look almost continuous, and it is easy to see that the market balance problem becomes almost a fixed-point problem. Khilnani and Tse ([5],[6]) reported that a contraction mapping algorithm can be used to solve a certain class of economic equilibrium problems. We show that combination trading belongs to this class of problems. We use the contraction mapping algorithm to obtain a nearly-optimal solution, where the price is not rounded.

We show how to obtain the optimal solution with a discrete price by solving a different fixed-point problem, namely a Kakutani fixed-point problem. Eaves ([3],[4]) reports an algorithm to solve Kakutani fixed-point problems which is based on path-following (homotopy). We implemented this algorithm.

Depending on the size of the problem, we recommend to solve market balance problems by applying in a first step the contraction mapping algorithm to obtain a nearly-optimal solution, and from there on by applying the path-following algorithm. This combined algorithm is much faster than the traditional linear programming approach, because the number of variables is much reduced: we aggregate all traders' limit quantities into demand and supply functions.

Exchange institutions compete against each other to attract trading volume, since some of them offer the same contracts. A critical feature of an exchange is to open the market very quickly at the beginning of the trading day, or after a trading halt. It is therefore important for an exchange to use a fast algorithm like ours.

\section{Model}

Notation: the sets $N, Z, Q, R$ are respectively the sets of nonnegative integers, integers, rational, and real numbers. The $R^{n}$ vector $e$ is the vector of ones. In the special case when each $i$-th component of the value of a $R^{m} \rightarrow R^{m}$ function $f$ depends on the $i$-th component $x_{i}$ of a variable $x$, we use the notation $f(x)$ for the vector value, and $f_{i}\left(x_{i}\right)$ for the component value. We write $\left[v_{i}\right]$ for the integral part of a real positive number $v_{i}$. When $v_{i}$ is negative, $\left[v_{i}\right]$ is the largest integer smaller than or equal to $v_{i}$. Finally, we write $[v]$ for a vector with integral components $\left[v_{i}\right]$.

In our model, traders place limit orders on an exchange, with a limit price, and a limit quantity. The exchange then sets a market price for each commodity, and allocates realized quantities to buyers and sellers against cash. Partial matching is allowed, meaning that buyers can receive any quantity up to the limit quantity, provided that the market price is not higher than their bids, and similarly for the sellers.

There are two kinds of commodities: primitive commodities, and combinations. Combinations are defined in terms of the quantity of each primitive commodity a combination buyer receives or delivers after his combination order is executed. We have $m$ primitive commodities and $n-m$ combinations. Each commodity $i=1 . . n$ 
is therefore defined by a $Q^{m}$ vector $a^{i}$, which represents the coefficients of each primitive commodity.

We collect the coefficient vectors of the combinations in an $Z^{m,(n-m)}$ matrix $A^{N}=\left(\begin{array}{llll}a^{m+1} & a^{m+2} & . . & a^{n}\end{array}\right)^{2}$, and define the matrix $A=\left(\begin{array}{ll}I & A^{N}\end{array}\right)$.

All traders have a linear utility function. We assume without loss of generality that each trader places only one order.

We can analyze traded quantities at three levels of trade aggregation. At the lowest level, we keep track of each deal between the traders. At the middle level, we aggregate each deal a trader took part in into his allocation. At the highest level, we group all traders with the same limit prices. We first present the middle level of trade aggregation, and then the highest. The lowest level will be needed only to prove theorem 1 .

\subsection{Middle level of trade aggregation}

On each commodity $i$, we have $N^{b}(i)$ buyers and $N^{s}(i)$ sellers. Each buyer $b=$ $1 . . N^{b}(i)$ of commodity $i$ places a limit order with limit price $\beta(i, b) \in Z$, and limit quantity $q^{b}(i, b) \in N$. Similarly, each seller $s=1 . . N^{s}(i)$ of commodity $i$ places a limit order with limit price $\sigma(s, i) \in Z$, and limit quantity $q^{s}(i, s) \in N$. The exchange institution then selects a price vector $p \in Z^{n}$ and an allocation $z^{b}(i, b) \geq 0$ for each buyer and $z^{s}(i, s) \leq 0$ for each seller. The value of the utility functions of the buyers and the sellers after trading will be:

$$
\begin{aligned}
U^{b}(i, b) & =\left(\beta(i, b)-p_{i}\right) z^{b}(i, b) \\
U^{s}(i, s) & =\left(\sigma(i, s)-p_{i}\right) z^{s}(i, s)
\end{aligned}
$$

The limit price constraints are, for all buyers and sellers:

$$
\begin{aligned}
& z^{b}(i, b)\left(p_{i}-\beta(i, b)\right) \leq 0 \\
& z^{s}(i, s)\left(p_{i}-\sigma(i, s)\right) \leq 0
\end{aligned}
$$

The limit quantity constraints are, for all buyers and sellers:

$$
\begin{array}{r}
0 \leq z^{b}(i, b) \leq q^{b}(i, b) \\
0 \leq-z^{s}(i, s) \leq q^{s}(i, s)
\end{array}
$$

The market clearing equations express the fact that combination orders can be matched against orders for primitive commodities:

$$
A\left(\begin{array}{c}
\sum_{b=1}^{N^{b}(1)} z^{b}(1, b)+\sum_{s=1}^{N^{s}(1)} z^{s}(1, s) \\
\ddot{.} \\
\sum_{b=1}^{N^{b}(n)} z^{b}(n, b)+\sum_{s=1}^{N^{s}(n)} z^{s}(n, s)
\end{array}\right)=0
$$




\subsection{Highest level of trade aggregation}

To determine the optimal price, we will see that the exchange institution needs only consider two $Z^{n} \rightarrow N^{n}$ functions: the aggregate demand $F^{b}$ and the aggregate supply $F^{s}$. For each commodity $i$, and $y_{i} \in Z$, we have:

$$
\begin{aligned}
& F_{i}^{b}\left(y_{i}\right)=\sum_{b=1}^{N^{b}(i)} q^{b}(i, b) 1\left(\beta(i, b) \geq y_{i}\right) \\
& F_{i}^{s}\left(y_{i}\right)=\sum_{s=1}^{N^{s}(i)} q^{s}(i, s) 1\left(\sigma(i, s) \leq y_{i}\right)
\end{aligned}
$$

, where $1(A)$ is the indicator function, i.e., it takes value 0 when $A$ is false, and 1 when $A$ is true.

We define $\left[y_{i}^{-}, y_{i}^{+}\right]$as the smallest interval that contains all buy and sell limit prices for commodity $i$, i.e., where the aggregate demand and supply vary.

In a similar fashion, we also aggregate the allocation into two $Z^{n} \rightarrow N^{n}$ functions: the aggregate buy quantity $f^{b}$, and the aggregate sell quantity $f^{s}$. For each commodity $i$, we have:

$$
\begin{aligned}
& f_{i}^{b}\left(y_{i}\right)=\sum_{b=1}^{N_{b}(i)} z^{b}(i, b) 1\left(\beta(i, b) \geq y_{i}\right) \\
& f_{i}^{s}\left(y_{i}\right)=-\sum_{s=1}^{N_{s}(i)} z^{s}(i, s) 1\left(\sigma(i, s) \leq y_{i}\right)
\end{aligned}
$$

In order to simplify the notation, we also define the allocated quantity for all traders that have the same limit price $y_{i}$ :

$$
\begin{aligned}
\Delta f_{i}^{b}\left(y_{i}\right) & =f_{i}^{b}\left(y_{i}\right)-f_{i}^{b}\left(y_{i}+1\right) \\
\Delta f_{i}^{s}\left(y_{i}\right) & =f_{i}^{s}\left(y_{i}\right)-f_{i}^{s}\left(y_{i}-1\right)
\end{aligned}
$$

\subsubsection{The combination trading problem}

The objective of the exchange institution is to maximize the utilitarian social welfare functional $z$, i.e., the sum of the profits of all traders. Obviously the results of this paper would still apply if the exchange institution chose a different social welfare functional. The decision variables of the exchange institution are the allocation $f^{b}(y), f^{s}(y)$, and the (integer) price $p$. The combination trading problem is: 


$$
\begin{array}{rlrl}
\max _{p, f^{b}(y), f^{s}(y)} z= & \sum_{y \in \mathcal{Y}} \sum_{i}\left[\Delta f_{i}^{b}\left(y_{i}\right)\left(y_{i}-p_{i}\right)\right. \\
& \left.+\Delta f_{i}^{s}\left(y_{i}\right)\left(p_{i}-y_{i}\right)\right] \\
f^{b}(y) & \geq 0 \quad \forall y \in \mathcal{Y} \\
f^{s}(y) & \geq 0 & \forall y \in \mathcal{Y} \\
f^{b}(y) & \leq F^{b}(y) & \forall y \in \mathcal{Y} \\
f^{s}(y) & \leq F^{s}(y) & \forall y \in \mathcal{Y} \\
\Delta f_{i}^{b}\left(y_{i}\right)\left(p_{i}-y_{i}\right) \leq 0 & \forall y \in \mathcal{Y}, i \\
\Delta f_{i}^{s}\left(y_{i}\right)\left(y_{i}-p_{i}\right) \leq 0 & \forall y \in \mathcal{Y}, i \\
A\left(f^{b}(p)-f^{s}(p)\right) & =0 & &
\end{array}
$$

where $\mathcal{Y}$ is a bounded set of all integer prices where the solution lies.

Relations (15) to (18) are implied by the limit quantity constraints (5) and (6). We will verify later that actually the solution to this model respects the limit quantity constraints, therefore it is not necessary to include these constraints in the formulation of the combination trading problem.

Relations (19) and (20) are the limit price constraints (3) and $(4)^{3}$, and relation (21) is the market clearing equation (7), at the aggregated level.

\subsubsection{The market balance problem}

One of the main results of the paper is to simplify the combination trading problem into another problem, the market balance problem. The market balance problem consists of determining $p^{B} \in \mathcal{Y}_{B}, f^{b}, f^{s}$ so that:

$$
\begin{aligned}
p= & \left(\begin{array}{ll}
p^{B} \quad p^{B} A^{N}
\end{array}\right) \\
\mathcal{Y}_{B}= & \cup_{i=1 . . n}\left\{\left\{p^{B} \in Z^{m} \mid y_{i}^{-} \leq a^{i} p^{B} \leq y_{i}^{+}\right\}\right. \\
& \left.\cap_{j=1 \ldots n}\left\{p^{B} \in Z^{m} \mid y_{j}^{-} \leq a^{j} p^{B} \leq y_{j}^{+}\right\}\right\} \\
F^{b}(p+e) \leq f^{b} \leq & F^{b}(p) \\
F^{s}(p-e) \leq f^{s} \leq & F^{s}(p) \\
A\left(f^{b}-f^{s}\right)= & 0
\end{aligned}
$$

It can be seen that $\mathcal{Y}_{B}$ is bounded and connected.

THEOREM 1 Let $\overline{p^{b}}, \overline{f^{b}}, \overline{f_{s}}$ solve the market balance problem. Then $p, f^{b}(y), f^{s}(y)$ solves the combination trading problem, where:

$$
\begin{aligned}
& p=\left(\begin{array}{ll}
\overline{p^{B}} & \overline{p^{B}} A^{N}
\end{array}\right) \\
& f^{b}(p)=\overline{f^{b}}
\end{aligned}
$$




$$
\begin{aligned}
& f^{b}(y)=F^{b}(y) \quad y_{i}<p_{i} \\
& f^{b}(y)=0 \quad y_{i}>p_{i} \\
& f^{s}(p)=\overline{f^{s}} \\
& f^{s}(y)=F^{s}(y) \quad y_{i}>p_{i} \\
& f^{s}(y)=0 \quad y_{i}<p_{i}
\end{aligned}
$$

PROOF see Appendix.

Relation (22) is a no-arbitrage relation. It states that the prices should be consistent ${ }^{4}$, i.e., that the prices of the combinations should be a combination of the price of the primitive commodities $p^{B}$. For instance, if the price of a future S\&P500 expiration July is $\$ 25$, and the price of a future $\mathrm{S} \& \mathrm{P} 500$ expiration June is $\$ 20$, then the optimal price of the spread should be $\$ 5$. Relation (23) is a simple consequence of price-consistency.

Inequalities (24) and (25) tell us that, for any commodity, all buyers (sellers) with a limit price strictly greater than (smaller than) the optimal price set by the exchange are allocated their entire limit quantity. The same property is a very well-known fact in simple markets without combinations.

\section{Algorithm}

Notation: for simplicity, we now write $p$ for the price of the primitive commodities instead of $p^{B}$.

We provide an algorithm to solve the market balance problem defined in the last section. From now, we call the latter discrete $(M B)$ problem, because the aggregate demand/supply is discrete. If there is a very large number of traders, and if we relax the integrality constraint on the limit prices, the aggregate demand/supply functions look almost continuous, and the discrete market balance problem is almost similar to the following continuous (MB) problem:

$$
A\left(F^{b}(p A)-F^{s}(p A)\right)=0
$$

An idea is therefore to approximate the discrete aggregate demand/ supply functions by a continuous function, solve the continuous (MB) problem to obtain an approximate real-valued solution $p^{r}$, and then search the exact solution $p$ in a neighborhood of $p^{r}$. We need therefore two algorithms, the continuous algorithm, and the discrete algorithm.

\subsection{Continuous algorithm}

For notational convenience we define the net demand function $G(y)=F^{b}(y)-$ $F^{s}(y)$. 
Let $G(y)$ be replaced by a piecewise linear function $G^{r}(y)$, such that $G_{i}^{r}\left(y_{i}\right)$ is strictly decreasing on $\left[y_{i}^{-}, y_{i}^{+}\right]$. We define $G_{i}^{r}\left(y_{i}\right)$ recursively in the interval $\left(y_{i}^{-}, y_{i}^{+}\right)$, starting from $y_{i}^{-}$, with $G_{i}^{r}\left(y_{i}^{-}\right)=F_{i}^{b}\left(y_{i}^{-}\right)-F_{i}^{s}\left(y_{i}^{-}\right)$. If $F_{i}^{b}\left(\left[y_{i}\right]+1\right)-F_{i}^{s}\left(\left[y_{i}\right]+1\right)<$ $F_{i}^{b}\left(\left[y_{i}\right]\right)-F_{i}^{s}\left(\left[y_{i}\right]\right)$ :

$$
\begin{aligned}
G_{i}^{r}\left(y_{i}\right)= & \left(1-y_{i}+\left[y_{i}\right]\right) G_{i}^{r}\left(\left[y_{i}\right]\right)+ \\
& \left(y_{i}-\left[y_{i}\right]\right)\left(F_{i}^{b}\left(\left[y_{i}\right]+1\right)-F_{i}^{s}\left(\left[y_{i}\right]+1\right)\right) \quad\left[y_{i}\right] \leq y_{i} \leq\left[y_{i}\right]+1
\end{aligned}
$$

Otherwise let $[z]$ be the maximal value of $t>\left[y_{i}\right]+1$ so that $F_{i}^{b}(t)-F_{i}^{s}(t)=$ $F_{i}^{b}\left(\left[y_{i}\right]\right)-F_{i}^{s}\left(\left[y_{i}\right]\right)$, then:

$$
G_{i}^{r}(x)=G_{i}^{r}\left(\left[y_{i}\right]\right)-\frac{1}{2} \frac{x-\left[y_{i}\right]}{[z]-\left[y_{i}\right]} \quad\left[y_{i}\right] \leq x \leq[z]
$$

Outside $\left(y_{i}^{-}, y_{i}^{+}\right)$, we define $G_{i}^{r}$ differently if $i$ is a primitive commodity or a combination. We introduce the superscripts ${ }^{B}$ as a mnemonic for primitive commodities ("basis"), and ${ }^{N}$ as a mnemonic for combinations ("nonbasis"). We redefine the inverse of $G^{B^{r}}$ and $G^{N^{r}}$ as:

$$
\begin{array}{rlrl}
G_{i}^{-B^{r}}\left(q_{i}\right) & =y_{i}^{-} & q_{i} \geq F_{i}^{b}\left(y_{i}^{-}\right)-F_{i}^{s}\left(y_{i}^{-}\right) \\
G_{i}^{-B^{r}}\left(q_{i}\right) & =y_{i}^{+} \quad q_{i} \leq F_{i}^{b}\left(y_{i}^{+}\right)-F_{i}^{s}\left(y_{i}^{+}\right) \\
G_{i}^{N^{r}}\left(y_{i}\right) & =F_{i}^{b}\left(y_{i}^{-}\right)-F^{s}\left(y_{i}^{-}\right) & y_{i} \leq y_{i}^{-} \\
G_{i}^{N^{r}}\left(y_{i}\right) & =F_{i}^{b}\left(y_{i}^{+}\right)-F^{s}\left(y_{i}^{+}\right) & y_{i} \geq y_{i}^{+}
\end{array}
$$

The continuous (MB) model is tantamount to finding the fixed point of $\mu(p)$, defined as:

$$
\mu(p)=G^{-B^{r}}\left(-A^{N} G^{N^{r}}\left(p A^{N}\right)\right)
$$

By definition, a function $\mu(p)$ is antitonic if, for any $p^{1}, p^{2}$ we have $\left(\mu\left(p^{1}\right)-\right.$ $\left.\mu\left(p^{2}\right)\right)\left(p^{1}-p^{2}\right) \leq 0$. Khilnani and Tse [5] report an algorithm that finds the fixed point of $\mu$, provided that $\mu$ is antitonic and Lipschitz continuous, with Lipschitz constant $\rho$.

THEOREM 2 If $\lambda<\frac{2}{1+\rho^{2}}$, the fixed point of $\mu$ can be found by iterating $p^{k+1}=$ $\phi\left(p^{k}\right)$, where $\phi(p)=(1-\lambda) p+\lambda \mu(p)$. The initial value $p^{0}$ is arbitrary.

PROOF $\mu(p)$ is antitonic because $G^{-B^{r}}$ and $G^{N^{r}}$ are decreasing. The rest of the proof goes along the lines of [5].

Note that this theorem guarantees the unicity of the solution of the continuous (MB) problem, which in turns shows that the domain of the prices that solve the discrete (MB) problem is bounded and connected. 


\subsection{Discrete algorithm}

In this section we show that the fixed point of a set-valued function $\mathcal{F}$ (to be defined later) is the solution of the discrete (MB) balance problem. We use the Eaves-Saigal algorithm to compute the fixed point of $\mathcal{F}$. Our discrete algorithm consists therefore of carefully defining $\mathcal{F}$ and then applying the Eaves-Saigal algorithm.

\subsubsection{The set-valued function $\mathcal{F}$}

Let $\mathcal{C}$ be the smallest parallelepiped that contains $\mathcal{Y}_{B}$. We call its maximal and minimal elements $p^{+}$and $p^{-}$. The value of our set-valued function $\mathcal{F}: \mathcal{C} \Rightarrow \mathcal{C}$ is the set $\left\{\bar{p} \in R^{m} \mid(\bar{p}, . . ., . .\right.$.$\left.) solves P_{1}(p)\right\}$, where $P_{1}(p)$ is a linear program that we define hereafter. It can be easily seen that $\mathcal{F}$ is convex-valued, and closed (by Berge's maximum theorem).

Before defining the linear program $P_{1}(p)$, we formulate the market balance problem slightly differently, using piecewise-linear demand and supply functions instead of discrete ones.

We need the following definitions:

$$
\begin{array}{rlrl}
F_{i}^{b^{\prime}}\left(y_{i}\right)= & F_{i}^{b}\left(\left[y_{i}\right]\right) & {\left[y_{i}\right] \leq y_{i} \leq\left[y_{i}\right]+\frac{2}{3}} \\
F_{i}^{b^{\prime}}\left(y_{i}\right)= & F_{i}^{b}\left(\left[y_{i}\right]\right)- & \\
& 3\left(y_{i}-\left[y_{i}\right]-\frac{2}{3}\right)\left(F_{i}^{b}\left(\left[y_{i}\right]\right)-F_{i}^{b}\left(\left[y_{i}\right]+1\right)\right) & & {\left[y_{i}\right]+\frac{2}{3} \leq y_{i} \leq\left[y_{i}\right]+1} \\
F_{i}^{s^{\prime}}\left(y_{i}\right)= & F_{i}^{s}\left(\left[y_{i}\right]\right) & & \\
F_{i}^{s^{\prime}}\left(y_{i}\right)= & F_{i}^{s}\left(\left[y_{i}\right]+\frac{1}{3} \leq y_{i} \leq\left[y_{i}\right]+1\right. \\
& 3\left(y_{i}-\left[y_{i}\right]+\frac{1}{3}\right)\left(F_{i}^{s}\left(\left[y_{i}\right]\right)-F_{i}^{s}\left(\left[y_{i}\right]-1\right)\right) & & {\left[y_{i}\right] \leq y_{i} \leq\left[y_{i}\right]+\frac{1}{3}} \\
D_{i i}^{b}\left(y_{i}\right)= & 3\left(y_{i}-\left[y_{i}\right]\right) & & {\left[y_{i}\right] \leq y_{i} \leq\left[y_{i}\right]+\frac{1}{3}} \\
& \left(F_{i}^{b^{\prime}}\left(\left[y_{i}\right]+1\right)-F_{i}^{b^{\prime}}\left(\left[y_{i}\right]\right)\right) & {\left[y_{i}\right]+\frac{1}{3} \leq y_{i} \leq\left[y_{i}\right]+1} \\
D_{i i}^{b}\left(y_{i}\right)= & F_{i}^{b^{\prime}}\left(\left[y_{i}\right]+1\right)-F_{i}^{b^{\prime}}\left(y_{i}\right) & {\left[y_{i}\right] \leq y_{i} \leq\left[y_{i}\right]+\frac{2}{3}} \\
D_{i i}^{s}\left(y_{i}\right)= & F_{i}^{s^{\prime}}\left(\left[y_{i}\right]\right)-F_{i}^{s^{\prime}}\left(y_{i}\right) & \\
D_{i i}^{s}\left(y_{i}\right)= & 3\left(\left[y_{i}\right]+1-y_{i}\right) & {\left[y_{i}\right]+\frac{2}{3} \leq y_{i} \leq\left[y_{i}\right]+1}
\end{array}
$$

The non-diagonal elements of $D^{s}$ and $D^{b}$ are set to zero. 
LEMMA Let $\left(f_{b}, f_{s}, p \in R^{m}\right)$ be a solution of the following modified market balance problem:

$$
\begin{aligned}
f^{b} & =F^{b^{\prime}}\left(A^{T} p\right)+D^{b}\left(A^{T} p\right) \tau^{b} \\
f^{s} & =F^{s^{\prime}}\left(A^{T} p\right)+D^{s}\left(A^{T} p\right) \tau^{s} \\
0 \leq \tau_{b} & \leq e \\
0 \leq \tau_{s} & \leq e
\end{aligned}
$$

Then $\left(f_{b}, f_{s},[p]\right)$ is a solution of the discrete market balance problem.

We now define the linear program $P_{1}(p)$ :

$$
\begin{aligned}
\max _{\substack{p^{\prime}, f b^{\prime}, s^{s^{\prime}}, \tau^{b^{\prime}}, \tau^{s^{\prime}}}} f^{b} B^{b^{-1}}\left(A^{T} p\right) f^{b^{\prime}} & +f^{s} B^{s^{-1}}\left(A^{T} p\right) f^{s^{\prime}} \\
f^{b^{\prime}}+B^{b}\left(A^{T} p\right) A^{T}\left(p^{\prime}-p\right) & \leq F^{b^{\prime}}\left(A^{T} p\right)+D^{b}\left(A^{T} p\right) \tau^{b} \\
f^{s^{\prime}}-B^{s}\left(A^{T} p\right) A^{T}\left(p^{\prime}-p\right) & \leq F^{s^{\prime}}\left(A^{T} p\right)+D^{s}\left(A^{T} p\right) \tau^{s} \\
A\left(f^{b^{\prime}}-f^{s^{\prime}}\right) & =0 \\
0 \leq \tau^{b^{\prime}} & \leq e \\
0 \leq \tau^{s^{\prime}} & \leq e
\end{aligned}
$$

The $R^{n}$ vectors $f^{b}$ and $f^{s}$ are any strictly positive vectors such that $A\left(f^{b}-f^{s}\right)=$ 0 . Obviously, $f^{b}=f^{s}=e$ would respect these conditions. The $R^{n} \rightarrow R^{n, n}$ (matrix) functions $B^{b}$ and $B^{s}$ have strictly positive and diagonal values. They can be carefully chosen so as to obtain better algorithmic performance. The EavesSaigal algorithm is much quicker if the initial simplex is small and contains the solution, which imposes constraints on the choice of $v^{k}$ (the $n+1$ vertices of the initial simplex), $B^{b}$ and $B^{s}$.

The vertices $v^{k}$ of the initial simplex are chosen so that:

$$
\begin{aligned}
& \frac{F_{i}^{b^{\prime}}\left(a^{i} v^{k}\right)-f^{b}}{a^{i} v^{k}-a^{i} p^{r}}>0 \\
& \frac{F_{i}^{s^{\prime}}\left(a^{i} v^{k}\right)-f^{s}}{a^{i} v^{k}-a^{i} p^{r}}<0
\end{aligned}
$$

,where again $p^{r}$ is the solution of the continuous market balance problem. For all vertices $v^{k}$ of the initial simplex we set

$$
\begin{aligned}
B_{i i}^{b}\left(A^{T} v^{k}\right) & =\frac{F_{i}^{b^{\prime}}\left(a^{i} v^{k}\right)-f^{b}}{a^{i} v^{k}-a^{i} p^{r}} \\
B_{i i}^{s}\left(A^{T} v^{k}\right) & =\frac{F_{i}^{s^{\prime}}\left(a^{i} v^{k}\right)-f^{s}}{a^{i} v^{k}-a^{i} p^{r}}
\end{aligned}
$$

so that the solution of $P_{1}\left(v^{k}\right)$ is $\left(p^{r}, f^{b}, f^{s}\right)$. For all other arguments, $B_{i i}^{b}$ and $B_{i i}^{s}$ should be linearly interpolated. 
THEOREM 3 All fixed points $p$ of $\mathcal{F}(p)$ are solutions of the modified market balance problem.

PROOF The matrix of the system of all linear equations that are part of the optimality conditions at a fixed point has full rank, by construction of $\mathcal{F}$. Solving this system we see that the multipliers $\lambda^{b}$ and $\lambda^{s}$ of inequalities (34) and (35) are equal to:

$$
\begin{aligned}
\lambda^{b} & =B^{b^{-1}} f^{b} \\
\lambda^{s} & =B^{s^{-1}} f^{s}
\end{aligned}
$$

Since $f^{b}, f^{s}>0$, then $\lambda^{b}, \lambda^{s}>0$. Therefore, at a solution $\left(\bar{p}, \bar{f}^{b}, \bar{f}^{s}, \bar{\tau}^{b}, \bar{\tau}^{s}\right)$ with $\bar{p}$ fixed point, constraints (34) and (35) are tight, i.e.:

$$
\begin{aligned}
& \bar{f}^{b}=F^{b^{\prime}}\left(A^{T} \bar{p}\right)+D^{b}\left(A^{T} \bar{p}\right) \bar{\tau}^{b} \\
& \bar{f}^{s}=F^{s^{\prime}}\left(A^{T} \bar{p}\right)+D^{s}\left(A^{T} \bar{p}\right) \bar{\tau}^{s}
\end{aligned}
$$

\subsubsection{The Eaves-Saigal algorithm}

We will not explain the Eaves-Saigal algorithm rigorously. The interested reader can consult [3][4]. It is a path-following (also called homotopy) method to determine the Kakutani fixed point of any closed and convex-valued set-valued function (also called multifunction in the literature).

Eaves-Saigal is a "multidimensional bisection algorithm". It starts from the full feasible domain as the initial simplex, subdivides it into subsimplices, chooses a subsimplex as the incumbent simplex (in such a way that the incumbent always contains the solution), and goes on subdividing until the incumbent simplex is small enough.

In the non-degenenerate case, a simplex $\sigma$ contains the solution if its vertex set is completely labeled, i.e., for all vertices $v^{k}$ and any $y^{k} \in \mathcal{F}\left(v^{k}\right)$ :

$$
\begin{aligned}
\sum_{k} \lambda_{k}\left(y^{k}-v^{k}\right) & =0 \\
\sum_{k} \lambda_{k} & =1 \quad \lambda_{k} \geq 0
\end{aligned}
$$

It is a remarkable fact that, if the algorithm takes care of degeneracy appropriately and starts from a completely labeled simplex of which no facet is completely labeled, then there always is a completely labeled subsimplex regardless of the subdivision.

The discrete market balance problem is particularly well suited to the EavesSaigal algorithm: 
- by construction of $\mathcal{F}$, more precisely of $B$, the initial simplex can be chosen much smaller than the smallest simplex containing $C$.

- the incumbent simplex does not need to be very small for the algorithm to terminate: it only needs to fit within the $m$-dimensional grid of integral prices to contain the solution of the market balance problem.

Therefore, the number of iterations between the initial and the terminal simplex will be small.

\subsection{Summary of the algorithm}

Bringing together the contraction mapping algorithm to solve the continuous (MB) model and the homotopy to solve the discrete (MB) model, we summarize hereafter our algorithm.

\section{Contraction mapping}

$1.1 k=0$; select $p_{0}^{r} \in \mathcal{Y}_{B}$

$1.2 \rho=\left\{\sum_{i} \frac{\left\{\sum_{k} \sum_{j}\left|A_{i j} A_{k j}\right| \max _{y} \frac{d G_{j}^{N^{r}}(y)}{d y}\right\}^{2}}{m \min _{y} \frac{d G_{i}^{B^{r}}(y)^{2}}{d y}}\right\}^{1 / 2}$

$1.3 \beta=\sqrt{\frac{1 / 2+\rho^{2}}{1+\rho^{2}}}$

$1.4 \lambda=\frac{1+\sqrt{\frac{1}{2}}}{1+\rho^{2}}$

1.5 repeat

$$
\begin{gathered}
k=k+1 \\
\quad p_{k}^{r}=(1-\lambda) p_{k-1}^{r}+\lambda G^{-B^{r}}\left(-A^{N} G^{N^{r}}\left(p_{k-1}^{r} A^{N}\right)\right) \\
\text { until }\left\|p_{k}^{r}-p_{k-1}^{r}\right\|<\frac{m}{1-\beta} \\
1.6 p^{r}=p_{k}^{r} ; \text { choose } f^{b}, f^{s}>0 \text { so that } f^{b}-f^{s}=G^{r}\left(A^{T} p^{r}\right)
\end{gathered}
$$

\section{Homotopy}

2.1 select an initial simplex with vertices $v^{k}$ such that $\frac{F_{i}^{b^{\prime}}\left(a^{i} v^{k}\right)-f^{b}}{a^{i} v^{k}-a^{i} p^{r}}>0$ and $\frac{F_{i}^{s^{\prime}}\left(a^{i} v^{k}\right)-f^{s}}{a^{i} v^{k}-a^{i} p^{r}}<0$

2.2 apply Eaves-Saigal algorithm on $\mathcal{F}$ until the incumbent simplex fits in a unit simplex $\sigma$

2.3 the solution $[p]$ is the highest price so that $[p] \leq y \quad \forall y \in \sigma$. 


\subsection{Complexity}

The only method that our (MB) model and algorithm can be compared to is the plain linear (PL) program defined in the appendix, which solves the combination trading problem at the lowest level of trade aggregation. Except in some very special cases, combination trading cannot be transformed into a small network flow problem.

Both methods are nonpolynomial, because they both rely on the simplex algorithm. However, the (MB) algorithm can be solved on average much quicker. To see that, we use the common approximation that the average number of iterations of the primal simplex method is proportional to the number of rows (see e.g. [2]), and each pivoting requires a number of operations equal to the number of elements of the simplex matrix.

The parameters involved in the complexity of the algorithm are $m$, the number of primitive commodities, $n>>m$, the total number of combinations, $p$, the average number of orders per combination, $q$, the length of a cube that contains all limit prices for all primitive commodities, and $r$, the ratio of the maximal slope of net

demand (of all net demand functions for combinations) over the minimal slope of net demand (of all net demand functions for primitive commodities).

Solving combination trading as a plain linear (PL) program would mean solving a linear program with $n$ rows and $n p^{m}$ columns (in the worst case where no coefficient $A_{i j}$ is equal to zero). Therefore, the (PL) method requires an average number of operations $T_{P L}$ equivalent to:

$$
T_{P L}=O\left(n^{3} p^{m}\right)
$$

To evaluate our method, we suppose that the average of the coefficients $A_{i j}$ is equal to one. Therefore the radius $\rho$ is $m n r(m(n-m) r$ to be accurate). Since $\rho$ is large, the parameter $\beta$ is approximately equal to $1-\frac{1}{4 \rho^{2}}$, and $\log \beta$ is approximately equal to $-\frac{1}{8 \rho^{2}}$

In the worst case, the norm of the difference between our two first price iterates in (1.5) will be $\sqrt{m} q / 2$. Since

$$
\left\|p_{k+1}^{r}-p_{k}^{r}\right\| \leq \beta\left\|p_{k}^{r}-p_{k-1}^{r}\right\|
$$

then

$$
\begin{aligned}
\left\|p_{k+1}^{r}-p_{k}^{r}\right\| & \leq \beta^{k}|| p_{1}^{r}-p_{0}^{r} \| \\
\frac{m}{1-\beta} & \leq \beta^{k} \sqrt{m} q / 2 \\
k & \geq \frac{\frac{1}{2} \log m-\log q}{-\log \beta} \\
k & \leq 8 m^{2} n^{2} r^{2} \log q
\end{aligned}
$$


is the maximum number of iterations of loop (1.5). Each loop requires $2 m n$ operations, so the contraction mapping has a total number of $O\left(m^{3} n^{3} r^{2} \log q\right)$ operations.

For the discrete algorithm the volume of the initial simplex is at most equal to $m^{m}$. What is the optimal number $x$ of subsimplices per simplex? The number of subdivisions to go from a simplex of size $S=m^{m}$ to a subsimplex of size 1 is $\log _{x} S$. The number of operations per subdivision being proportional to the number of subsimplices, the optimal $x$ minimizes $x \log _{x} S$, therefore $x=m$ is a good choice, and $\log _{m} S=\log _{m} m^{m}=m$ is the number of subdivisions. For each subdivision:

- the linear program $P_{1}(p)$ has to be solved once; our subdivisions are made in such a way that each new vertex will be integral most of the time, so that $D^{b}\left(A^{T} p\right)=D^{s}\left(A^{T} p\right)=0$, and therefore the dimension of $P_{1}(p)$ is reduced; this step needs $O\left(n^{3}\right)$ operations

- we then have to solve between 1 and $m$ systems of $m$ equations with $m$ unknowns, which can be achieved in at worst $O\left(m^{4}\right)$ operations.

Therefore the homotopy requires a total time of $O\left(m^{5}+m n^{3}\right)$ operations and the average number of operations $T_{M B}$ of the (MB) algorithm is:

$$
T_{M B}=O\left(m^{3} n^{3} r^{2} \log q+m^{5}+m n^{3}\right)
$$

$\underline{\text { Example }}$

If $m=10, n=100, p=100, r=100$, and $q=100$, then $T_{M B} \approx 10^{12}$ and $T_{L P} \approx 10^{26}$.

\subsection{Numerical results}

In order to validate the claim that our fixed-point algorithm is superior to plain linear programming (LP) we implemented both algorithms on the same computer (PC Pentium) and the same language (Visual Basic).

Table 1 shows that the fixed point algorithm is indeed faster than plain (LP) when implemented on a standard class of combination trading problems: 2 primitive commodities, and 1 combination. We tried 10 different problems, each one with a different number of distinct limit prices. As expected, computation time for plain (LP) grows very fast with the number of distinct limit prices. It does not for our fixed point algorithm. 
Table 1. Speed comparison fixed point algorithm versus plain PL

\begin{tabular}{|c|c|c|}
\hline \multirow{2}{*}{$\begin{array}{l}\text { Number of distinct } \\
\text { limit prices }\end{array}$} & \multicolumn{2}{|c|}{ Algorithm speed [s] } \\
\hline & Plain LP & Fixed Point \\
\hline 9 & 2.0 & 1.0 \\
\hline 12 & 3.7 & 1.1 \\
\hline 15 & 8.7 & 0.7 \\
\hline 18 & 8.1 & 1.3 \\
\hline 21 & 49.3 & 1.4 \\
\hline 24 & 42.0 & 1.4 \\
\hline 27 & 98.5 & 1.5 \\
\hline 30 & 242.6 & 1.5 \\
\hline 33 & 236.6 & 1.5 \\
\hline 36 & 52312.3 & 1.6 \\
\hline
\end{tabular}

The experiments reported in table 1 corresponded to test cases where the fixed point algorithm would start far away from the solution, i.e., at the highest price lower than the solution where the aggregate demand and supply curves for each primitive commodity are flat. In table 2 , we repeat the last experiment of table 1 (with 36 distinct limit prices) and vary the initial point; a distance of $100 \%$ in the table means that the initial price is the highest price lower than the solution where the curves are flat. As expected, the closer the initial point to the solution, the faster the algorithm.

Table 2. Speed of fixed point algorithm with different initial prices

\begin{tabular}{|c|c|}
\hline Distance initial price to solution & Speed [s] \\
\hline $100 \%$ & 1.60 \\
$90 \%$ & 1.54 \\
$80 \%$ & 1.49 \\
$70 \%$ & 1.45 \\
$60 \%$ & 1.41 \\
$50 \%$ & 1.34 \\
$40 \%$ & 1.29 \\
$30 \%$ & 1.19 \\
$20 \%$ & 1.03 \\
$10 \%$ & 0.81 \\
0 & 0.14 \\
\hline
\end{tabular}

\section{Conclusion}

The two main results of this paper are the proof that consistent prices are optimal, and the existence of an algorithm to compute the optimal price and allocation 
quickly. In our model the variables have been aggregated, so that the dimension of the problem is greatly reduced compared to the traditional linear programming approach. For large problems our algorithm is very efficient, because of its two main features, price-consistency and trade aggregation.

We also believe that our model could add further insight to many issues of the market microstructure literature, such as the existence of market manipulation strategies.

\section{Appendix: proof of theorem 1}

In this appendix we prove only that consistent prices are optimal; i.e. $p^{N}=p^{B} A^{N}$. The proof that the solution of $(\mathrm{MB})$ respects the optimality conditions is available upon request.

At the lowest level of trade aggregation, the combination trading problem becomes:

$$
\begin{gathered}
\max _{x^{N}, x^{C} \geq 0} \gamma^{N} x^{N}+\gamma^{C} x^{C} \\
\sum_{s=1}^{N^{s}(i)} x^{N}(i, b, s)+\sum_{w \in W} w_{i}^{b} \sum_{d \in D_{i, b}^{b}} x^{C}(w, d) \leq q^{b}(i, b) \quad \forall(i, b) \\
\sum_{b=1}^{N^{b}(i)} x^{N}(i, b, s)+\sum_{w \in W} w_{i}^{s} \sum_{d \in D_{i, s}^{s}} x^{C}(w, d) \leq q^{s}(i, s) \quad \forall(i, s)
\end{gathered}
$$

The left-handside of (55) is nothing else than the buy allocation $z^{b}(i, b)$, decomposed into the "normal deals" allocated quantities $x^{N}$ made with the sellers of the same commodity $i$, and the "combination deals" allocated quantities $x^{C}$ made with buyers and sellers of different commodities. Each deal brings the following profit per quantity:

$$
\begin{aligned}
\gamma^{N}(i, b, s) & =\beta(i, b)-\sigma(i, s) \\
\gamma^{C}(w, d) & =\sum_{i=1}^{n} w_{i}^{b} \beta\left(i, d_{i}^{b}\right)-w_{i}^{s} \sigma\left(i, d_{i}^{s}\right)
\end{aligned}
$$

The set $W$ is the set of all vectors $w=\left(\begin{array}{ll}{ }^{b} & w^{s}\end{array}\right) \in N^{2 n}$ such that $A w^{b}-A w^{s}=$ $w^{b} w^{s}=0$, and no vector $w^{k}$ is the multiple of another vector $w^{l}$. In other words, $w$ represents the weight of each commodity in a particular type of combination deal.

$D_{i, b}^{b}$ is the set of all possible dealer groups $d=\left(\begin{array}{ll}d^{b} & d^{s}\end{array}\right)$ that take part in a particular deal involving $i$-th commodity buyer $b$. For instance, $d_{j}^{b}=2$ means that the second buyer of commodity 2 is part of the dealer group $d . D_{i, s}^{s}$ is defined analogously. By convention, only one trader per commodity takes part in a deal. 
The dual of the combination trading problem is:

$$
\begin{aligned}
\min _{u, v \geq 0} q^{b} u+q^{s} v & \\
u(i, b)+v(i, s) & \geq \gamma^{N}(i, b, s) \quad \forall i, b, s \\
\sum_{i=1}^{n} w_{i}^{b} u\left(i, d_{i}^{b}\right)+w_{i}^{s} v\left(i, d_{i}^{s}\right) & \geq \gamma^{C}(w, d) \quad \forall w, d
\end{aligned}
$$

By complementary slackness, we know that if the corresponding realized quantity $\overline{x^{N}(i, b, s)}$ and $\overline{x^{C}(w, d)}$ are strictly positive, then the optimal $\bar{u}$ and $\bar{v}$ satisfy:

$$
\begin{aligned}
\overline{u(i, b)}+\overline{v(j, s)} & =\beta(i, b)-\sigma(i, s) \\
\sum_{i=1}^{n} w_{i}^{b} \overline{u\left(i, d_{i}^{b}\right)}+w_{i}^{s} \overline{v\left(i, d_{i}^{s}\right)} & =\sum_{i=1}^{n} w_{i}^{b} \beta\left(i, d_{i}^{b}\right)-w_{i}^{s} \sigma\left(i, d_{i}^{s}\right)
\end{aligned}
$$

The strong duality theorem of linear programming implies that the optimal value of the objective of the primal is equal to the optimal value of the objective of the dual. With this in mind, we will identify, as in [10], $\overline{u(i, b)}$ as the optimal profit per share of $i$-trader $b$. Therefore there exists a price $p$ so that, for all $(i, b, s)$ with $\overline{z^{b}(i, b)}$ and $\overline{z^{s}(i, s)}$ strictly positive,

$$
p_{i}=\beta(i, b)-\overline{u(i, b)}=\sigma(i, s)+\overline{v(i, s)}
$$

Therefore

$$
\begin{aligned}
& \overline{u(i, b)}=\beta(i, b)-p_{i} \\
& \overline{v(i, s)}=p_{i}-\sigma(i, s)
\end{aligned}
$$

Inserting (65) and (66) into (63):

$$
\sum_{i=1}^{n} w_{i}^{b}\left(\beta(i, b)-p_{i}\right)+w_{i}^{s}\left(p_{i}-\sigma(i, s)\right)=\sum_{i=1}^{n} \beta(i, b) w_{i}^{b}-\sigma(i, s) w_{i}^{s}
$$

i.e.,

$$
\sum_{i=1}^{n}\left(w_{i}^{b}-w_{i}^{s}\right) p_{i}=0
$$

We define a new vector $t$ by $t=w^{b}-w^{s}$. We decompose $t ; p$ into $t^{B} ; p^{B}$ for the primitive instruments and $t^{N} ; p^{N}$ for the combinations. Relation (68) becomes $p^{B} t^{B}+p^{N} t^{N}=0$ and has to be satisfied for any integral $t$ so that $t^{B}+A^{N} t^{N}=0$. Therefore $p^{N}$ has to be equal to $p^{B} A^{N}$, and optimal prices have to be consistent. 


\section{Notes}

1. Suppose a trader wants to buy stock $A$ only if the price difference between index $B$ and stock $A$ is more than $\$ 1$. In a combination trading exchange, this would be achieved by placing 2 orders:

- one combination order to buy $A$ and sell $B$ only if the price difference is less than $\$ 1$

- $\quad$ one market order to buy $B$

The only problem is that markets may not clear if traders place too many market orders. We believe however that the conditions for a combination trading exchange to clear are less stringent than the conditions for the conditioned mechanism described in [11] to converge.

2. To ease the exposition we assume now, by rescaling, that all coefficient vectors of the combinations are integral.

3. Consequently, the optimal solution should respect $\Delta f_{i}^{b}\left(y_{i}\right)=0$ for $y_{i}<p_{i}$ and $\Delta f_{i}^{s}\left(y_{i}\right)=0$ if $y_{i}>p_{i}$.

4. Our definition of consistency is more general than the definition of consistency in [1]. Trades $f^{b}-f^{s}$ in [2] are consistent when they respect the relation $\left(f^{b}-f^{s}\right) v=0$ for some vector $v$.

\section{References}

1. A. Beja and N. Hakansson. Dynamic market processes and the rewards to up-to-date information, Journal of Finance, 32, no 2, 291-304, 1977.

2. G.B. Dantzig. Linear programming and extensions. Princeton University Press, 1963.

3. B.C. Eaves. Lecture notes in equilibrium programming. Stanford University, Spring 1992.

4. B.C. Eaves and R. Saigal. Homotopies for computation of fixed points on unbounded regions, Mathematical Programming, 3, no 2, 1972.

5. A. Khilnani, A. and E. Tse. A fixed point algorithm with economic applications, Journal of Economic Dynamics and Control, 9, 127-137, 1985.

6. A. Khilnani and E. Tse. A note on the radius of convergence of the USA algorithm, Journal of Economic Dynamics and Control, 13, 313-316, 1989.

7. A. Madhavan. Trading mechanisms in securities markets, Journal of Finance, 47, no. 2, 607-641, 1992.

8. R. Miller. The design of decentralized auction mechanisms that coordinate continuous trade in synthetic securities. Journal of Economic Dynamics and Control, 14, 237-253, 1990.

9. H. Schellhorn. Optimization of combination trading with limit orders. Doctoral dissertation, UCLA, unpublished, 1995.

10. L.S. Shapley and M. Shubik. The assignment game I: the core, International Journal of Game Theory, 1, 111-130, 1971/2.

11. A. Wohl. The feasibility of an index-contingent trading mechanism, Management Science, 43, no 1, 112-121, 1997. 


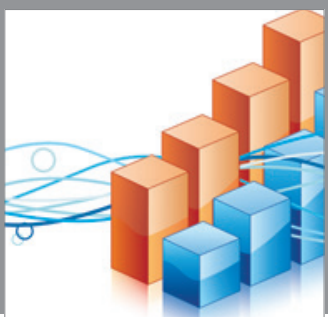

Advances in

Operations Research

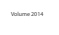

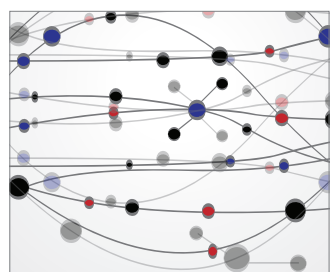

\section{The Scientific} World Journal
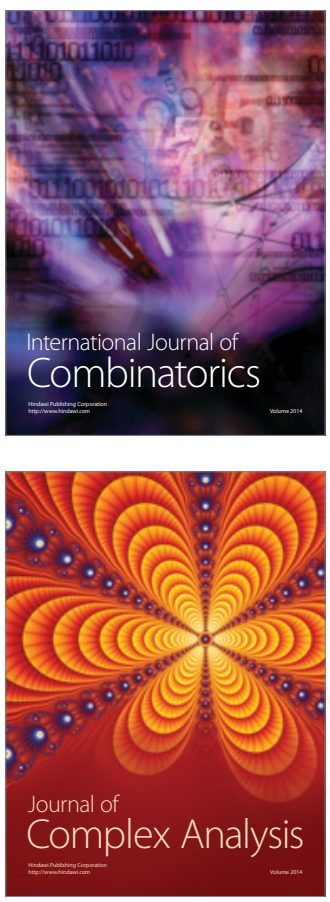

International Journal of

Mathematics and

Mathematical

Sciences
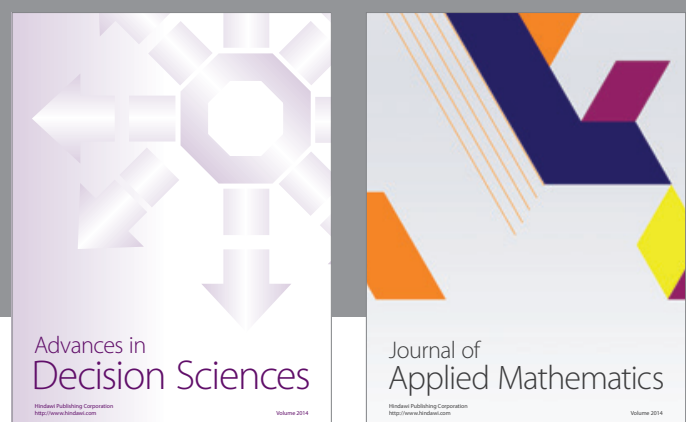

Journal of

Applied Mathematics
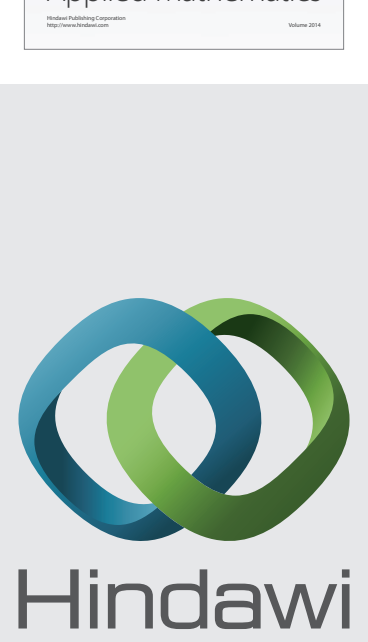

Submit your manuscripts at http://www.hindawi.com
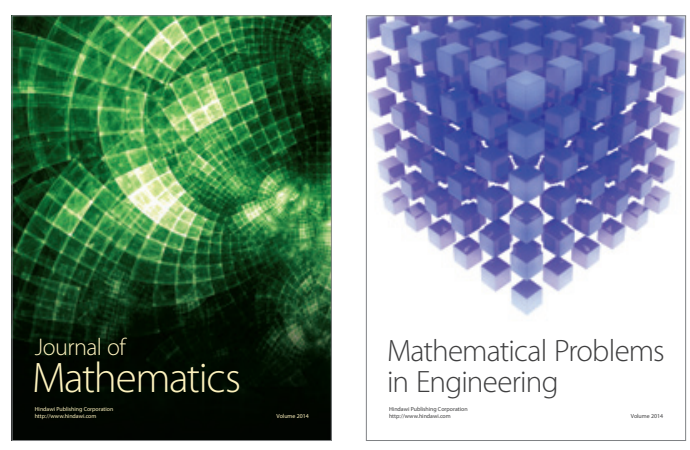

Mathematical Problems in Engineering
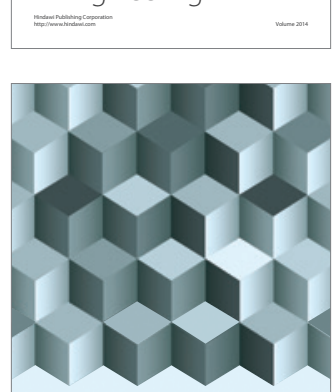

Journal of

Function Spaces
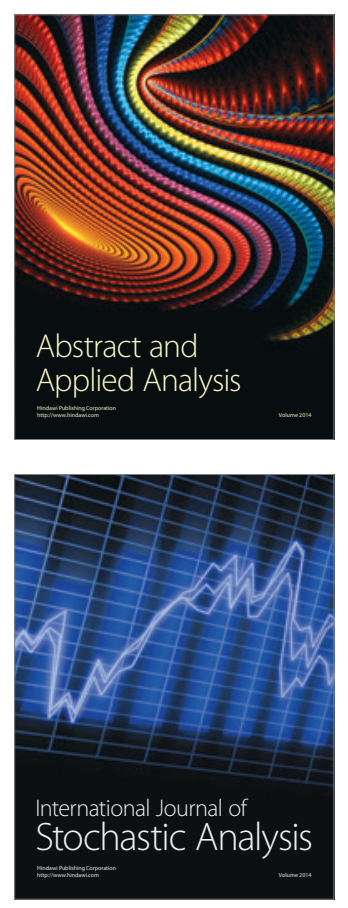

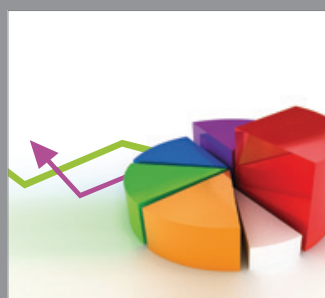

ournal of

Probability and Statistics

Promensencen
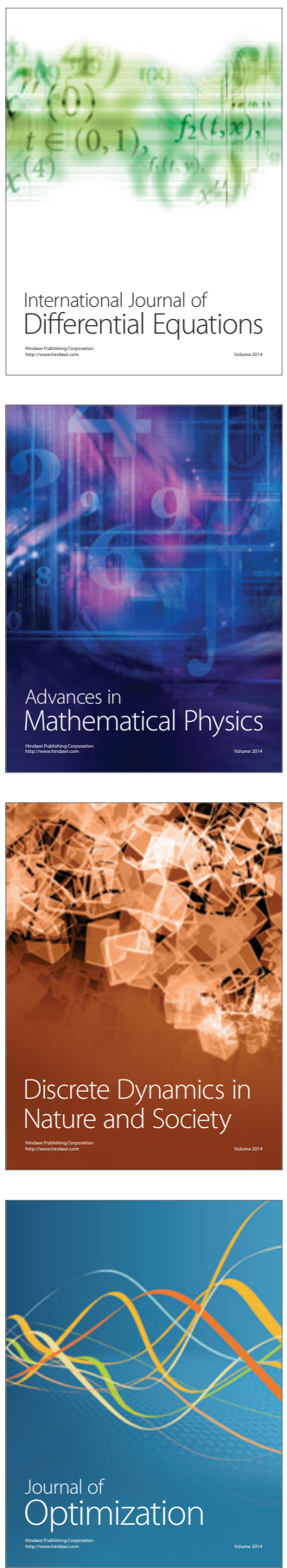